\title{
Die Psychidenfauna der Republik Zypern (Lepidoptera: Psychidae)
}

\author{
Mit 6 Figuren und 1 Karte
}

Michael WeIdLICH ${ }^{1}$

${ }^{1}$ Lindenallee 11, 15898 Neißemünde, OT Ratzdorf, Deutschland. - dr.michael.weidlich@gmail.com

Published on 2015-06-30

\section{Zusammenfassung}

In der vorliegenden Arbeit wird der Kenntnistand zur Psychidenfauna der Republik Zypern mitgeteilt. Aktuell liegen Nachweise von insgesamt 6 Arten dieser Familie vor. Eine neue Art, Eumasia cyprianella spec. nov. wird hierin beschrieben.

Der Verfasser weilte in den Jahren 1998, 1999, 2012 und 2014 auf der Insel. Erste Ergebnisse der Aufsammlungen wurden bereits 2002 mit der Erstbeschreibung von Pseudobankesia aphroditae Weidlich \& Henderickx, 2002 veröffentlicht. Auf morphologische und genetische (MtDNA) Details sowie auf die Verbreitung von Pachythelia villosella (Ochsenheimer, 1810) wird näher eingegangen und das Taxon P. villosella quadratica De FrejnA, 1983 wird diskutiert. Weitere Angaben zur Biologie und Ökologie der Psychiden der Republik Zypern vervollständigen die Arbeit.

\section{Key words}

Republic of Cyprus, faunistic review, Eumasia cyprianella spec. nov., Psychidae, Lepidoptera, biology, morphology, history

\section{Summary}

In the present paper the knowledge of the bagworm moth of the Republic of Cyprus is discussed. Currently 6 species of this family are known from Cyprus. Also a new species Eumasia cyprianella spec. nov. is described herein. The author visited the island in the years 1998, 1999, 2012 and 2014. First results of the collecting trips were published in 2002, including the first description of Pseudobankesia aphroditae Weidlich \& Henderickx, 2002.

Morphological and cytochemical (mtDNA) characteristics are addressed and the distribution of Pachythelia villosella (Ochsenheimer, 1910) is discussed. The taxon P. villosella quadratica De Freina, 1983 is reviewed. Further particulars on the biology and ecology of the Psychidae of the Republic of Cyprus Psychidae complete the work.

\section{Einleitung}

Zypern ist mit $9251 \mathrm{~km}^{2}$ Fläche nach Sizilien und Sardinien die drittgrößte Mittelmeerinsel und befindet sich im östlichsten Teil des Mittelmeerraumes. Der Olympos ist mit $1952 \mathrm{~m} \mathrm{NN}$ die höchste Erhebung auf der Insel und Bestandteil des Tróodos-Gebirges. Weitere Informationen zur Geologie, Geographie, zum Klima,

zur Flora und Vegetation der Insel finden sich in einer Übersicht bei MAKRIS (2003: 18-27).

Seit 1974 existiert auf dem südlichen Teil der Insel die „Republik von Zypern“, die seit 2004 Mitglied der Europäischen Union ist. Auf dem nördlichen Teil befin- 
det sich die international nicht anerkannte „Türkische Republik Nordzypern“.

Die ersten Angaben von zypriotischen Schmetterlingen stammen von Dr. Theodor Kotschy aus dem Jahre 1841, die aber zur damaligen Zeit nicht publiziert wurden (vergl. ReBeL, 1939: 492). Im Jahre 1853 entsandte Julius LEDERER den Insektensammler Franz ZACH zur Insel. Dessen Ergebnisse, die aus der Umgebung von Lárnaka, vom Nordrand der Insel und Stavrovouni stammen, sind dann durch LEDERER (1855: 183) veröffentlicht worden. Aus dieser Zeit stammt auch der erste Nachweis einer zypriotischen Psychide und wurde als Pachythelia villosella (OCHSEnheimer, 1810) veröffentlicht. Auch spätere Arbeiten, die, wenn sie sich überhaupt zur Psychidenfauna äussern, nennen immer nur dieses Taxon (vergl. Rebel, 1916: 109; 1939: 543, WimMER, 1985: 57; LEWANDOWSKI, 2000: 377; LEWANDOWSKI \& FISCHER, 2002: 272; FISCHER \& LEWANDOWSKI, 2003: 301; LEWANDOWSKI \& MAKRIs, 2006: 167). Weitere Psychidensäcke, die bei LEWANDOWSKI \& MAKRIs (2006: 165, 167) erwähnt und abgebildet sind, stellten sich als Säcke einer Coleophoridenart heraus. Die Herkunft der Angaben von Eumasia parietariella (HEYDENREICH, 1851) sowie Apterona helicoidella (VAllot, 1827) bei Karsholt \& VAN Nieukerken (2013) http://en.wikipedia.org/wiki/List_of_Lepidoptera_ of_Cyprus (abgerufen am 03.11.2014) ließen sich nicht ermitteln und können somit nicht eindeutig der Republik Zypern zugeordnet werden. Eine erste zusammenfassende Arbeit über alle bekannten Schmetterlingsnachweise von Zypern legte ReBel (1939) vor. Darauf aufbauend sind gegenwärtig für fast alle Schmetterlingsfamilien/-gruppen ausführliche, neuere Bearbeitungen bekannt. So beispielsweise für die Mikrolepidoptera (ARENBERger, 1994), Geometridae (Hausmann, 1995; Fischer \& LeWANDOWsKi, 2010), Rhopalocera und Hesperiidae (Makris, 2003), Noctuidae (LewANDowski \& Fischer, 2004) sowie für die Bombyces \& Sphinges die oben erwähnten Arbeiten von FISCHER und LEwANDowsKI.

Der Autor besuchte vom 06.-12.03.1998, gemeinsam mit RALF WeSER, 24.-31.03.1999, 12.-19.03.2012 jeweils alleine und 04.-18.06.2014 gemeinsam mit seiner Frau Dr. Ilona Weser die Republik Zypern. Während dieser Reisen wurden hauptsächlich Psychiden beobachtet. Als Beifänge sind auch Arten anderer Lepidopterenfamilien beobachtet und gesammelt worden.

Bei den ersten Aufsammlungen ist bereits 1998 eine neue Psychiden-Art entdeckt worden, Pseudobankesia aphroditae WeIDLICH \& HeNDERICKx, 2002, und es wurden auch schon einige Beifänge publiziert (vergl. Arenberger \& Wimmer, 2003).

Eine Untersuchung der Psychidenfauna, besonders unter Berücksichtigung moderner DNA-Analysen fehlte bisher und bildet einen Gegenstand der vorliegenden Arbeit. Insgesamt gesehen ist die Psychidenfauna noch nicht sehr umfassend bekannt und aus dem türkischen Teil Zyperns sind bisher noch überhaupt keine Funde veröffentlicht worden.

\section{Die Psychidenfundorte der Republik Zypern (= Fundortverzeichnis)}

Die administrative Gliederung der beiden Republiken besteht aus 6 Regierungsbezirken bzw. Distrikten. Es sind dies Páphos, Nicosia, Lemesós, Lárnaka, Famagusta und Kyrenia. Durch die Teilung der Insel wurden die Distriktbereiche von Nicosia und Lárnaka getrennt und durch beide Republiken gesondert verwaltet. Famagusta und Kyrenia liegen in der Türkischen Republik Nordzypern. Beide Republiken sind durch eine Demarkationslinie voneinander getrennt.

Alle bisher aus der Literatur bekannten Fundorte werden im Verzeichnis geführt. Stammen Nachweise des Autors ebenfalls von diesen publizierten Fundorten, wird dies zusätzlich erwähnt. Alle übrigen angegebenen Fundorte gehen auf Nachweise des Verfassers zurück.

Die Auflistung der Fundorte in den einzelnen Regierungsbezirken erfolgt chronologisch und in deren karthografischer Darstellung wird jeweils dem jüngsten Nachweis der Vorrang gegeben. Die Lage aller Fundorte ist in der Karte 1 aufgeführt.

\section{Provinz Páfos (= Páphos):}

Fundort 1: Páfos (= Páphos) (Wimmer, 1985: 57; LEWANDOWSKI \& FISCHER, 2002: 272; WEIDLICH)

Fundort 2: Neofytos, 400 m NN (= Neóphytos) (LEwANDOWSKI \& FISCHER, 2002: 272)

Fundort 3: Neo Chõrio, $140 \mathrm{~m} \mathrm{NN}$

Fundort 4: Kedares 0,5 km SE, $500 \mathrm{~m} \mathrm{NN}$

Fundort 5: Tróodos-Gebirge, Umg. Agios Nikolaos, 2-8 km N, Fluß Diarizos, 500-550 m NN (WEIdLICH \& HeNDERICKx, 2002: 504, 505)

Fundort 6: Akamas, Umg. Akoursos E, Bäder des Adonis, $250 \mathrm{~m} \mathrm{NN}$ (Weidlich \& HendeRICKX, 2002: 504, 505)

Fundort 7: Umg. Tsada ca. 3 km N, 450 m NN (WEIDLICH \& HENDERICKX, 2002: 504, 505)

Fundort 8: Tróodos-Gebirge, Umg. Páno Panagiá, $2 \mathrm{~km}$ N Kannaviou, $450 \mathrm{~m}$ NN (WEIDLICH \& HENDERICKX, 2002: 504)

Fundort 9: Tróodos-Gebirge, Moumourus/Argaki tis Agias, Umg. Stavros S, 900 m NN und Umg. Stavros $1 \mathrm{~km} \mathrm{~N}, 950 \mathrm{~m} \mathrm{NN}$ (Weidlich \& HENDERICKX, 2002: 504, 505)

Fundort 10: Tróodos-Gebirge, Moumourus/Argaki tis Agias, Umg. Stavros $8-13 \mathrm{~km} \mathrm{~S}$, 600-750 m NN (WEIDLICH \& HENDERICKX, 2002: 504, 505) 
Fundort 11: Polis, ca. $20 \mathrm{~m} \mathrm{NN}$ (LewANdowski, 2000: 377)

Fundort 12: Umg. Peyia SE, Hotel Corallia Beach ca. $2 \mathrm{~km} \mathrm{E}, \mathrm{ca} .10 \mathrm{~m} \mathrm{NN}$

Fundort 13: Tróodos-Gebirge, Umg. Páno Panagiá 8 km NE, Karka-Fluß Vrysia, 450 m NN (WeIDLICH \& HeNDERICKX, 2002: 504, 505)

Fundort 14: Petra tou Rõmiou (Aphrodites Geburtsplatz), $25 \mathrm{~m} \mathrm{NN}$

Fundort 15: Umg. Neo Chõrio, Bath of Aphroditae, ca. $50 \mathrm{~m} \mathrm{NN}$ (Weidlich \& HeNderickx, 2002: 504)

Fundort 16: Latsi, ca. 10-20 m NN (LewANDowski \& FISCHER, 2002: 272)

Fundort 17: Umg. Mandria $1 \mathrm{~km} \mathrm{~N}$ bei Páfos E, $10 \mathrm{~m} \mathrm{NN}$

Fundort 18: Umg. Kouklia 2 km SE, 15 m NN

Fundort 19: Umg. Kouklia ca. 2 km NW, 100 m NN

Fundort 20: Umg. Kidasi 1 km SW, 250 m NN

Fundort 21: Umg. Goudi E, $90 \mathrm{~m} \mathrm{NN}$

Fundort 22: Nea Dimmata, $30 \mathrm{~m} \mathrm{NN}$

Fundort 23: Umg. Panagia Galaterousa 0,5 km NE (= Panagia Galatarka), $50 \mathrm{~m} \mathrm{NN}$

Fundort 24: Pomos, $5 \mathrm{~m} \mathrm{NN}$

Fundort 25: Umg. Peyia NE, Avakas Gorge, 50 m NN

Provinz Lefkosia (=Nicosia):

Fundort 26: Kakopetria, 700 m NN (Lewandowski \& FisCHER, 2002: 272)

Fundort 27: Tróodos-Gebirge, Mouti tis Primithias, Umg. Mylikouri S, 350-980 m NN
Fundort 28: Tróodos-Gebirge, Marathasa, KykkoMonastery 5-7 km E, 900-1000 m NN (WEIDLICH \& HENDERICKX, 2002: 504, 505)

Fundort 29: Tróodos-Gebirge, Marathasa, Kykko-Monastery $2 \mathrm{~km} \mathrm{~S}, 550-650 \mathrm{~m} \mathrm{NN}$ (WEIDLICH \& HENDERICKX, 2002: 504, 505)

Fundort 30: Tróodos-Gebirge, Marathasa, Kykko-Monastery $9 \mathrm{~km} \mathrm{E}, 900 \mathrm{~m} \mathrm{NN}$ (WEIDLICH \& HENDERICKX, 2002: 504, 505)

Fundort 31: Tróodos-Gebirge, Tripolis Nature Reserve/ Zedernschlucht, $1100 \mathrm{~m} \mathrm{NN}$ (WEIDLICH \& HENDERICKX, 2002: 504, 505, 512)

Fundort 32: Umg. Apliki ca. 1 km E, 750 m

Fundort 33: Tróodos-Gebirge, Fluß Diarizos, Agios Nikolaos 8-10 km N, 500-550 m NN (WEIDLICH \& HENDERICKx, 2002: 504, 505)

Fundort 34: Umg. Lythrodontea E, 400 m NN (= Lythrodontas)

Fundort 35: Tróodos-Gebirge, Prodromus S, 1400 m NN

Fundort 36: Tróodos-Gebirge, Evrichou N, 420 m NN

Fundort 37: Koronia Südhänge bei Kato Koutrafes SW, $250 \mathrm{~m} \mathrm{NN}$

Fundort 38: Tróodos-Gebirge, Umg. Lagoudera N, Panagia tou Araka, $1040 \mathrm{~m} \mathrm{NN}$

Fundort 39: Tróodos-Gebirge, Palaichori S, 950 m NN

\section{Provinz Lemesós (=Limassol):}

Fundort 40: Agia Fyla, ca. 160 m NN (Fischer \& LewANDOWSKI, 2003: 301)

Fundort 41: Umg. Koilani 1 km S, 750 m NN

Fundort 42: Umg. Parekklisia S und N, 50-220 m NN

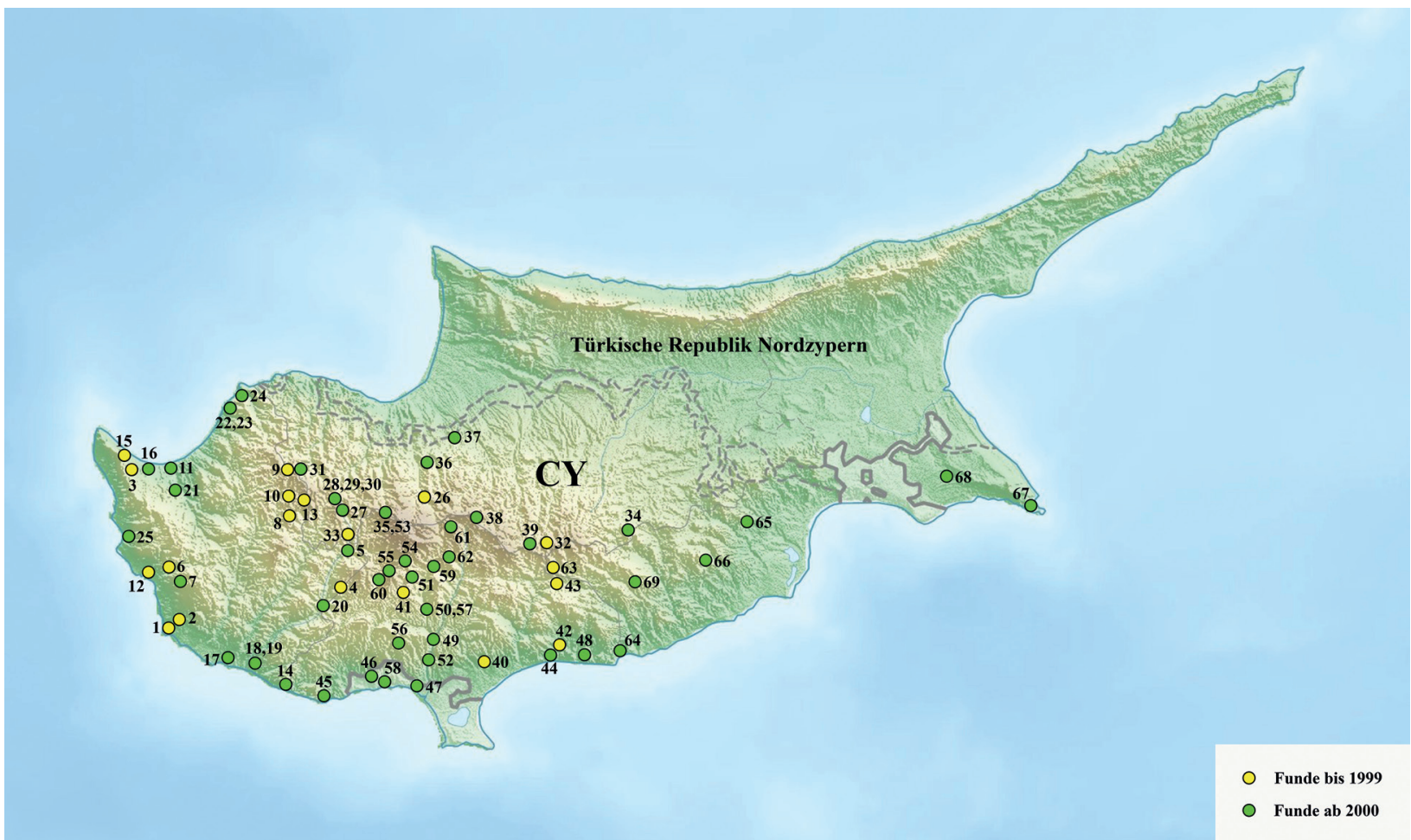

Karte 1: Lage der zypriotischen Psychidenfundorte mit Beobachtungszeiträumen (verändert nach http://de.wikipedia.org/wiki/ Zypern $\sigma^{\star}$ ediaviewer/File:Cyprus_relief_location_map.jpg). 
Fundort 43: Umg. Kellaki ca. 1,5 km N, 540 m NN

Fundort 44: Amathus, ca. $130 \mathrm{~m} \mathrm{NN}$ (LewANDOWsKi \& MAKRIS, 2006: 167)

Fundort 45: Umg. Pissouri SE, Hotel Columbia Beach, $5 \mathrm{~m} \mathrm{NN}$

Fundort 46: Umg. Pissouri E, Paramali, $70 \mathrm{~m} \mathrm{NN}$ (Episcopi Cantonment)

Fundort 47: Apollon Hylátis Heiligtum, Antikes Stadion und Antikes Koúrion, 60-110 m NN (= Curium) (Episcopi Cantonment)

Fundort 48: Umg. Limassol E, Autobahnabfahrt Moni, $30 \mathrm{~m} \mathrm{NN}$

Fundort 49: Umg. Alassa W, $290 \mathrm{~m} \mathrm{NN}$

Fundort 50: Umg. Monagri S, $490 \mathrm{~m} \mathrm{NN}$

Fundort 51: Tróodos-Gebirge, Umg. Pera Pedi NW, $830 \mathrm{~m} \mathrm{NN}$

Fundort 52: Umg. Souni SE, $320 \mathrm{~m} \mathrm{NN}$

Fundort 53: Lemithou, $1080 \mathrm{~m} \mathrm{NN}$ (BOLDT inhttp.//lepiforum.de/2_forum.pl?md=read;id=94829)

Fundort 54: Tróodos-Gebirge, Umg. Páno Plátres, $1180 \mathrm{~m} \mathrm{NN}$

Fundort 55: Mandria W, $840 \mathrm{~m} \mathrm{NN}$

Fundort 56: Pano Kivides W, $500 \mathrm{~m} \mathrm{NN}$

Fundort 57: Dhoros E, $490 \mathrm{~m} \mathrm{NN}$

Fundort 58: Umg. Episcopi W, $30 \mathrm{~m} \mathrm{NN}$ (Episcopi Cantonment)

Fundort 59: Umg. Saittas ca. 1,5 km NE, 700 m NN

Fundort 60: Omodus N, $850 \mathrm{~m} \mathrm{NN}$

Fundort 61: Kyperounta S, $1.190 \mathrm{~m} \mathrm{NN}$

Fundort 62: Umg. Pelentri W, 880 m NN

\section{Provinz Lárnaka:}

Fundort 63: Umg. Odou ca. 0,5 km SE, $660 \mathrm{~m} \mathrm{NN}$

Fundort 64: Umg. Governor's Beach N, Autobahnabfahrt, $40 \mathrm{~m} \mathrm{NN}$

Fundort 65: Umg. Kalo Chõrio W, 90 m NN

Fundort 66: Umg. Stavrovouni Monestery, $500 \mathrm{~m} \mathrm{NN}$

Fundort 67: Cavo Greko, $60 \mathrm{~m} \mathrm{NN}$

Fundort 68: Liopetri N, $45 \mathrm{~m} \mathrm{NN}$

Fundort 69: Umg. Pano Lefkara S, 600 m NN

\section{Systematisch-faunistisches Verzeichnis der} Psychiden der Republik Zypern

Es werden alle bekannten Literaturquellen ausgewertet und das vorliegende Material detailliert beschrieben. In der Beschreibung der einzelnen Arten werden die Fundorte nur in der Kurzform ohne Entfernungs- und Höhenangaben genannt. Bei der Neubeschreibung von Eumasia cyprianella spec. nov. wird hiervon eine Ausnahme gemacht und der vollständige Fundort aufgeführt.

Folgende Abkürzungen finden Verwendung: e.l. = ex larva, e.p. = ex pupa, i.A. = in Anzahl (3-9 Exemplare), i.M. = in Menge (ab 10 Exemplare).

\section{Psychidae BoISDUVAL, 1829}

Incertae sedis

\section{Eumasia cyprianella spec. nov.}

Holotypus: $\sigma^{\top}$, e.l. 05.07.2014: Asia occidentalis, Republik Zypern, Tróodos-Gebirge, Páno Plátres, 1180 m NN, leg. Dr. M. Weidlich (Fig. 1). Er befindet sich in coll. Museum für Naturkunde des Leibniz Institutes in Berlin.

Derivatio nominis: Die Art wird nach der Insel Zypern benannt, auf der sie offensichtlich endemisch vorkommt.

Paratypen (die gezüchteten Imagines jeweils mit Sack und Puppenhülle):

Männchen: $1 \sigma^{\top}$ e.l. 30.05.2012, $2 \sigma^{*}$ e.l. 01.06.2012, $1 \sigma^{\star}$ e.l. 01.06.2012, 5 ơ e.l. Mitte 06.2012: Umg. Tsada ca. 3 km N, $450 \mathrm{~m} \mathrm{NN}$; 1 o e.l. 08.07.2014, 7 o e.l. 07.-10.07.2014, 3 ơ e.l. 11.07.2014, 6 o 12.-18.07.2014: Tróodos-Gebirge, Umg. Páno Plátres, 1180 m NN; alles leg. WEIDLICH.

Weibchen: 1 우 e.l. 30.05.2012, 2 우 e.l. 01.06.2012, 4 우 e.l. 02.06.2012, 1 우 e.l. 03.06.2012, 2 우 e.l. Mitte 06.2012: Umg. Tsada ca. $3 \mathrm{~km} \mathrm{N,} 450 \mathrm{~m} \mathrm{NN}$; 3 ㅇ e.l. 05.07.2014 (Fig. 1), 2 우 e.l. 07.-10.07.2014, 1 우 e.l. 11.07.2014, 13 우 12.-18.07.2014: Tróodos-Gebirge, Umg. Páno Plátres, 1180 m NN; 1 ㅇ e.l. 27.06.2014: Tróodos-Gebirge, Marathasa, Kykko-Monastery $7 \mathrm{~km} \mathrm{E,} 900 \mathrm{~m} \mathrm{NN}$; alles leg. WEIDLICH.

Säcke: 4 Säcke, 08.03.1998, 4 Säcke, 10.03.1998: Tróodos-Gebirge, Marathasa, Kykko-Monastery $6 \mathrm{~km} \mathrm{E,}$ 950 m NN; 1 Sack, 09.03.1998: Akamas, Umg. Akoursos E, Bäder des Adonis, 250 m NN; 1 Sack 11.03.1998; Tróodos-Gebirge, Moumourus/Argaki tis Agias, Umg. Stavros S, 900 m NN; 3 Säcke, 11.03.1998: Umg. Stavros, 8-13 km S; 1 Sack 27.03.1999: Tróodos-Gebirge, Moumourus/Argaki tis Agias, Umg. Stavros $10 \mathrm{~km} \mathrm{~S}$ 600 m NN; 3 Säcke 09.03.1998, 6 Säcke 12.03.1998, 11 Säcke 25.03.1999, 5 Säcke 30.03.1999, 5 Säcke 14.03. 2012, 12 Säcke 17.03.2012, 4 Säcke 09.06.2014:Umg. Tsada ca. $3 \mathrm{~km} \mathrm{~N}, 450 \mathrm{~m} \mathrm{NN}$; 2 Säcke 27.03.1999: TróodosGebirge, Umg. Páno Panagiá, $2 \mathrm{~km} \mathrm{~N}$ Kannaviou, 450 m NN; 97 Säcke 04.-16.06.2014: Tróodos-Gebirge, Umg. Páno Plátres, 1180 m NN; 2 Säcke 13.06.2014: Umg. Episcopi W, $30 \mathrm{~m} \mathrm{NN}$ (Episcopi Cantonment); 2 Säcke 08.06.2014: Tróodos-Gebirge, Prodromus S, 1.400 m NN; 1 Sack 11.06.2014: Tróodos-Gebirge, Marathasa, Kykko-Monastery $7 \mathrm{~km} \mathrm{E,} 900 \mathrm{~m} \mathrm{NN}$; 1 Sack 15.06.2014 Tróodos-Gebirge, Umg. Lagoudera N, Panagia tou Araka, 1040 m NN; alles leg. WEIDLICH.

Das Typenmaterial umfasst 27 Männchen mit Säcken, 30 Weibchen mit Säcken und 160 Säcke sowie 1 Sack mit Raupe und stammt von dreizehn Lokalitäten (Karte 1). 


\section{Beschreibung:}

Diagnose: Beide Geschlechter geflügelt, Männchen mit einer Flügelspanne von 8,3 bis $8,7 \mathrm{~mm}$ und Weibchen mit eine Flügelspanne von 8,7 bis $10,0 \mathrm{~mm}$. Kopf dicht mit langen, meist bräunlichen Haaren bedeckt. Augen schwarz, rund, ohne Ocellen. Der Augenabstand beträgt etwa das Doppelte des Augendurchmessers, Labialpalpen relativ lang, dreigliederig. Fühler kurz und sie erreichen etwa $1 / 3$ bis $1 / 2$ der Länge des Vorderflügelcostalrandes. Sie sind bei beiden Geschlechtern dicht mit grauen Schuppen bedeckt und bei den Männchen tritt eine ventral ausgerichtete Bewimperung hinzu. Fühlergliederzahl mit Scapus und Pedicellus 25 bis 29.

Vorderflügelfärbung dunkelbraun, versehen mit sehr unregelmäßig verteilter, dunkelgelber Punktierung, die selten zu schmalen Bändern zusammenfließt. Diskoidalfleck nicht erkennbar. Die Deckschuppen sind breit, meist sechszackig (Schuppenklasse 5 bis 6 nach SAUTER, 1956: 498). Vorderflügeladerung mit 10 Zelladern, wovon 7 der offenen Diskoidalzelle entspringen, Medius 2 und 3 entspringen aus einem Punkt. Hinterflügel dunkelgrau, Deckschuppen breit, zumeist Schuppenklasse 5. Fransenschuppen sehr lang, etwas heller, meist mit leicht gelblichem Ton und von lanzettlicher Form, meist zweizackig.

Hinterflügeladerung mit 7 erkennbaren Adern, wovon 5 aus der offenen Diskoidalzelle entspringen. Thorax mit unterschiedlichen Haaren und Schuppen versehen. Abdomen dicht mit breiten, grauen Schuppen bedeckt. 7. Abdominalsegment des Weibchens kranzförmig mit

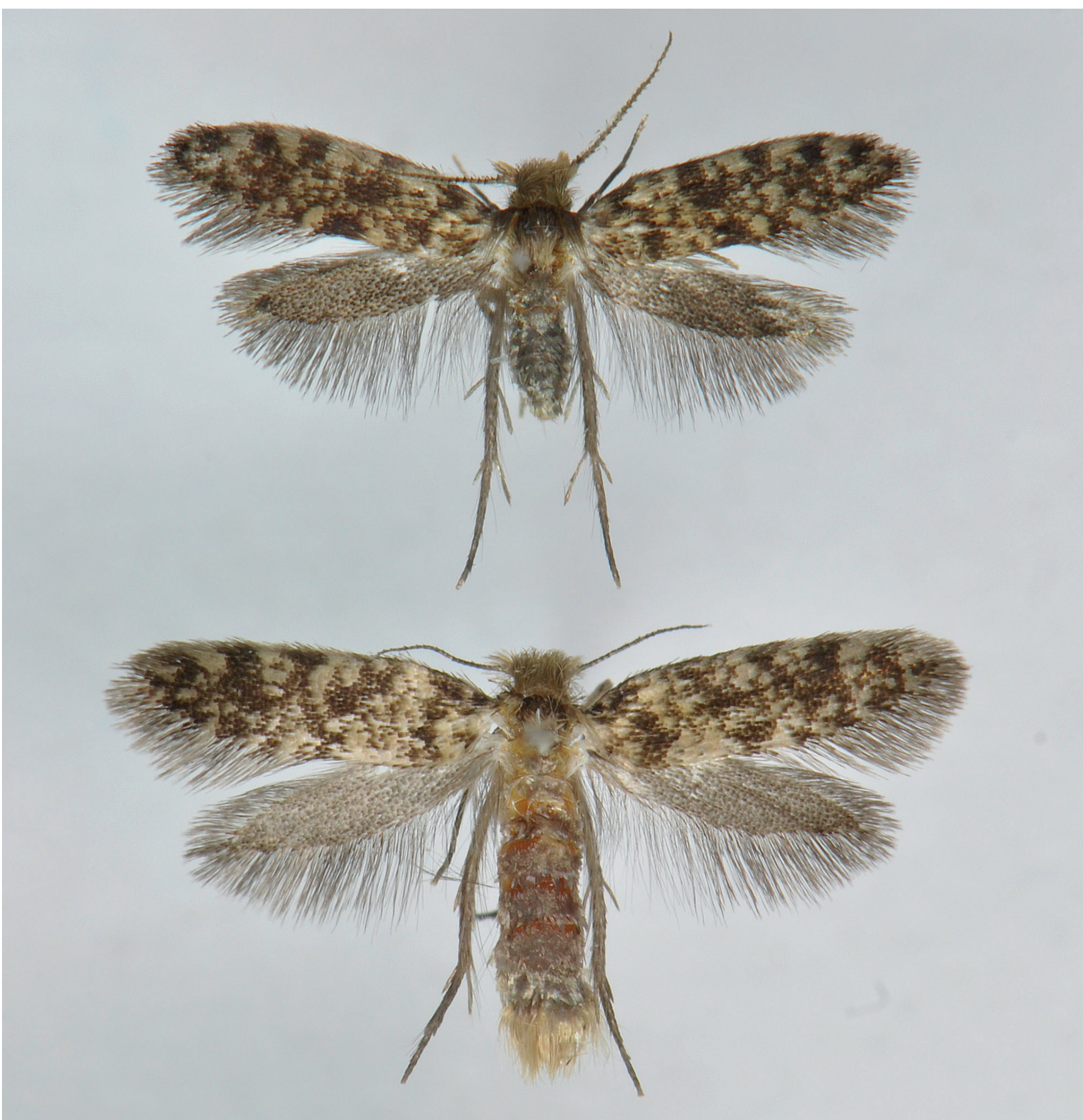

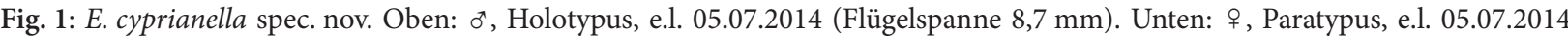
(Flügelspanne 10,0 mm), Asia occidentalis, Republik Zypern, Tróodos-Gebirge, Páno Plátres, 1180 m NN, leg. WeidLICH. (Foto: M. WEIDLICH). 
langen, geraden, gelblichen Haaren bedeckt. Darunter befinden sich dorsal und ventral etwas kürzere, gewellte, braune Haaren. Der Ovipositor überragt die Gesamtkörperlänge oftmals deutlich. Vordertibien ohne Epiphyse aber mit einem langen Borstenpinsel versehen, Mitteltibien mit einem Spornpaar, Hintertibien mit zwei Spornpaaren. Alle Beine mit 5 Tarsengliedern.

Genitalapparat Eumasia - typisch, $0^{*}$ - Valven kurz, erreichen nicht das distale Ende des Tegumens, Clasper kurz und spitz und stark sklerotisiert, Sacculus bildet mit dem Vinculum ein spitzes Dreieck. Aedaeagus lang und leicht s-förmig gebogen. Seine Länge beträgt mehr als 4/5 der Länge des Genitals.

Ebenfalls typisch + - Antevaginalplatte bedornt aus einem schmalen Band, welches proximal deutlich eingebuchtet ist.

Sack: langgestreckt, rundlich und bestehend aus kleinen Detritusteilchen. An der vorderen Öffnung sind Chitinteilchen, Pflanzenteile oder größerer Detritus befestigt, welche dem Sack sein typisches Aussehen geben.

Die Länge der Säcke beträgt 9 bis 13 mm, ihr Durchmesser liegt um 1,5 bis $2 \mathrm{~mm}$. Die Gesamtbreite kann je nach Anzahl und Größe der angesponnenen Partikel durchaus bis $5 \mathrm{~mm}$ betragen. Insgesamt sind die weiblichen Säcke meistens etwas größer. Ihre Färbung reicht von hellgelbgrau (Fig. 2) bis mittelbraun.

Differentialdiagnose: Von der Gattung Eumasia Chrétien, 1904 sind bisher 13 Arten beschrieben worden. Aus der paläarktischen Fauna sind es E. parietariella (Heydenreich, 1851), E. communita Meyrick,

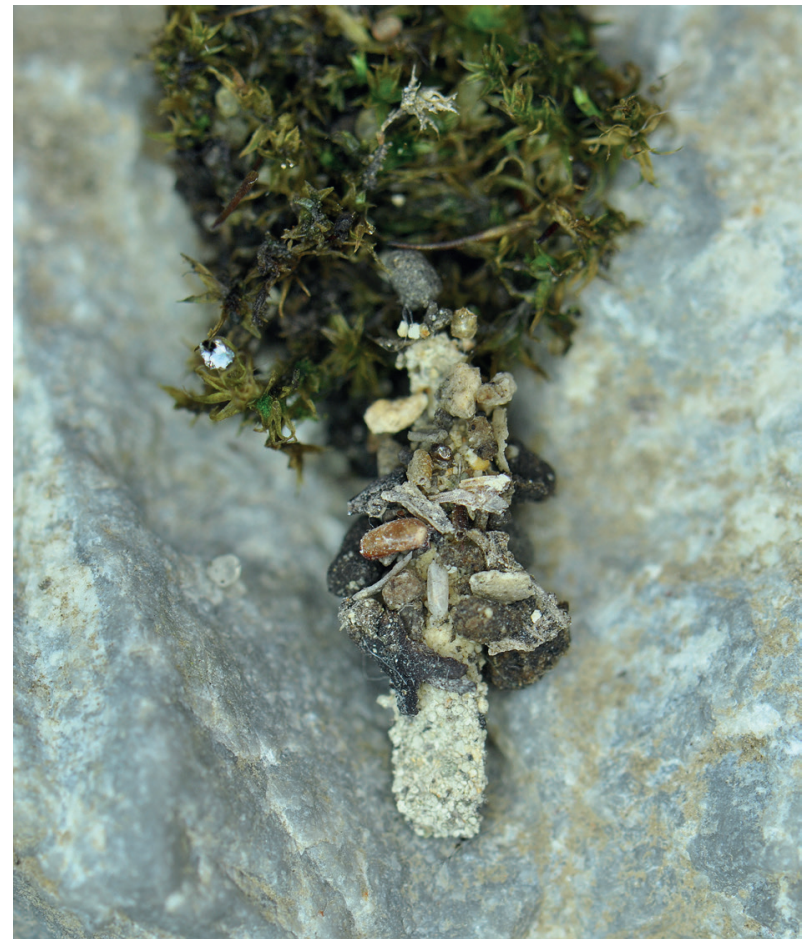

Fig. 2: Sackparatypus, 15.06.2014 (Sacklänge 9,8 mm), Asia occidentalis, Republik Zypern, Tróodos-Gebirge, Umg. Lagoudera N, Panagia tou Araka, 1040 m, leg. WeidLich.(Foto: M. WEIDLICH).
1928, E. montanella Solyanikov, 1986, E. libanotica Zagulajev, 1996, E. brunella HättensChWiler, 1998, E. muscella Saigusa \& Sugimoto, 2005 und E. viridilichenella Saigusa \& Sugimoto, 2005. Sechs weitere Arten kommen auf Ceylon, den Malediven und in Indien vor (vergl. SoвCZyк, 2011: 128-131). E. montanella ist im Tienschan Mittealasiens verbreitet und E. muscella und E. viridilichenella sind aus Japan beschrieben worden. E. communita ist aus Nordafrika (Marokko - Hoher Atlas) und E. brunella aus Spanien sowie Portugal bekannt.

E. parietariella ist im Mittelmeerraum und nördlich bis nach Mitteleuropa verbreitet. Die Angaben aus der Türkei (Bithynien) nach STAUdinger \& Rebel (1901: 241) gehören wahrscheinlich einer anderen Eumasia-Art an (vergl. WEIDLICH, 2010: 131).

Die von Zypern aus nächsten, gesicherten E. parietariella-Angaben liegen aus Griechenland vor. Aus dem ostmediterranen Raum wurde E. libanotica nach einem Männchen von ZAGULAJEV (1996: 117) beschrieben.

Habituell steht die neue Art der E. brunella am nächsten. Vor allem in der bräunlichen Flügelfärbung sind Ähnlichkeiten vorhanden (vergl. HäTteNSCHWILER 1998: 267), E. cyprianella ist jedoch größer. E. parietariella ist dagegen im Durchschnitt noch etwas größer, in der Grundfärbung hellgelblich und E. libanotica ist weißgelblich und ebenfalls etwas größer $(10 \mathrm{~mm}$, $0^{x}$-Holotypus). Die Ausprägung der Zeichnungselemente auf den Vorderflügeln der Imagines ist sehr variabel und sie lässt sich zur näheren Determination kaum verwenden. E. libanotica weist eine geschlossenen Diskoidalzelle auf, die bei E. parietariella, E. brunella und E. cyprianella offen ist. Die Länge des Borstenpinsels am Vorderbein ist bei E. libanotica deutlich kürzer und entspricht bei E. brunella etwa der Vordertibienlänge. Bei der neuen Art überragt sie die Vordertibienlänge. Besonders deutliche Unterschiede bestehen beim männlichen Genital. Die besondere Form und Länge des Aedaeagus bei E. cyprianella sowie auch bei E. libanotica unterscheidet sich von E. parietariella (gestreckt) und E. brunella (kurz nur $2 / 3$ der Genitallänge).

Bei den Weibchen bestehen Unterschiede an der Form des Dornenfeldes auf der Antevaginalplatte. Sie verläuft bei E. parietariella und E. brunella proximal konkav (vergl. HätTenschWiler, 1998: Fig. 6c und d), bei E. cyprianella ist sie deutlich lobenförmig ausgebildet (ähnlich, aber nicht so breit wie bei E. testata (vergl. HäTtenSCHWILER, 1998: Fig. 6f). Alle bisherigen E. parietariella Angaben für Zypern (siehe oben) beziehen sich auf die neue Art.

Biologie und Ökologie: E. cyprianella spec.nov. ist univoltin und die erwachsenen Larven (letztes Stadium) wurden hauptsächlich im Juni gefunden. Die Lebensräume bilden feuchte Felswände mit reichlichem Moos-, Flechten- und Algenbewuchs. Sie sind meist schattig bis halbschattig gelegen und zumeist nördlich ausgerichtet. 
Dort leben die Raupen in Vertiefungen, unter Vorsprüngen und Spalten ziemlich versteckt, wo sie sich auch zur Verpuppung anspinnen. Die Weiterzucht gelang mit Gabe von verschiedenen Moosen, Flechten, Algen und Pflanzenresten.

Der geologische Untergrund der Habitate erscheint zweitrangig, vornehmlich wurde die Art auf Kalksteinen, aber auch auf Andesit und Metamorphiten (Tróodos-Gebirge) beobachtet. E. cyprianella spec. nov. besiedelt Höhenlagen von 30 (Episcopi) bis $1.400 \mathrm{~m} \mathrm{NN}$ (Tróodos-Gebirge). Bei der Zucht schlüpften die Imagines vornehmlich in den Vormittagsstunden.

\section{Taleporiinae HERRICH-SCHÄFFER, 1857}

\section{Taleporiini TUTT, 1900 \\ 2. Pseudobankesia aphroditae WeIDLICH \& HENDERICKX, 2002}

Literatur: Bath of Aphrodite; Stavros 8-13 km S; Tripolis Nature Reserve, Zedernschlucht; Tsada; Mylikouri Kykko-Monastery; Diarizos/Agios Nikolaos; Vrysia/ Panõ Panagia; Kannaviou; Bäder des Adonis (ausführliche Angaben siehe Typenserie bei WeIdLICH \& HENDERICKX, 2002: 504, 505).

Untersuchtes Material: 19 Säcke 14., 17. und 18.03.2012: Tsada; 10 Säcke 16.03.2012: Kykko-Monastery 5 km E; 1 Sack 16.03.2012: Koilani; 5 Säcke, 18.03.2012 und 1 Sack 11.06.2014: Tripolis Nature Reserve/Zedernschlucht; 2 Säcke, 18.03.2012: Mylikouri, 5-6 km S, $500 \mathrm{~m} \mathrm{NN}$; 2 Säcke, 18.03.2012: Agios Nikolaos, alles leg. WEIDLICH.

Diskussion: Neben den bereits publizierten Fundorten in der Erstbeschreibung kommen nunmehr zwei weitere Fundorte hinzu. Die Art ist oft mit Eumasia cyprianella spec. nov. vergesellschaftet.

\section{Typhoniinae LEDERER, 1853}

Typhoniini LEDERER, 1853

\section{Typhonia spec.}

Neu für Zypern!

Untersuchtes Material: 1 Sack 17.03.2012: Tsada; leg. WEIDLICH.

Diskussion: Aus Zypern war bisher noch keine Typhonia -Art bekannt. Aufgrund des einzelnen Sackfundes kann keine nähere Bestimmung erfolgen. Die geographisch am nächsten vorkommenden Typhonia-Arten stammen aus Palästina (T. paulusella (CARADJA, 1921)), Türkei (T. punctata Herrich-SCHÄFFER, 1854) sowie vom grie- chischen Peloponnes (T. christenseni HäTtensCHWILER, 2000). Von der türkischen Riviera (Pamphylien, Kilikien) liegen weiterhin diverse Sack- und Raupenfunde vor (WEIDLICH ab 2002, unveröff.). Eine sehr ähnliche Struktur der Säcke, vor allem in den ersten Entwicklungsstadien, zeigen weiterhin die Arten der Gattung Eochorica REBEL, 1940. Nach bisherigen Kenntnissen sind diese aber auf Mazedonien, Bulgarien, Griechenland und Albanien? sowie auf die Westtürkei beschränkt (vergl. DANIEL, 1964: 55; WITT, 1984: 48-49; WEIDLICH, 1989: 10; 2013a: 335; De Freina, 1994: 328). Ein Vorkommen auf Zypern ist deshalb wenig wahrscheinlich.

Psychinae BoISDUVAL, 1840

Psychini BoIsDUVAL, 1829

4. Luffia cf. lapidella (GoEZE, 1763)

Neu für Zypern!

Literatur: 9 Säcke 14./17.03.2012: Tsada, (WEIDLICH, 2013b: 166).

Untersuchtes Material: 3 Säcke 09.06.2014: Tsada, leg. WEIDLICH.

Diskussion: Aus dem eingetragenen Material konnten bisher keine Imagines erzielt werden. Deshalb ist die Determination noch nicht gesichert. Es kann jedoch durchaus angenommen werden, dass es sich hier um die im mediterranen Raum verbreitet vorkommende L. lapidella handelt.

\section{Oiketicinae HERRICH-SCHÄFFER, 1855}

Acanthopsychini TutT, 1900

\section{Pachythelia villosella (OCHSENHEIMER, 1810)}

Literatur: Cypern (LEDERER, 1855: 183; REBEL, 1916: 109; 1939: 543); Paphos (Wimmer, 1985: 57; LeWANDOWSKI \& Fischer, 2002: 272); Polis (LeWANDOWSKI, 2000: 377); Latsi, Neofytos, Kakopetria, Paphos (LewAndowski \& Fischer, 2002: 272); Agia Fyla (Fischer \& LEWANDOWSKI, 2003: 301); Amathous (LEWANDOWSKI \& MAKRIS, 2006: 167).

Untersuchtes Material: 1 Sack 08.03.1998: Kedares; 43 Säcke 09.03.1998, 7 o o o e.l. 13.04.-30.04.1998; 12 Säcke 25.03.1999, 83 Säcke 29.03.1999, 3 Säcke 30.03. 1999, 16 o o o $^{\text {e }}$ e.1. 03.-19.05.1999, 1 क e.l. 12.05.1999, 38 Säcke 14.03.2012; 47 Säcke 17.03.2012, 28 Säcke 18.03. 2012, 7 o $^{\star} o^{\star}$ e.l. 30.05.-12.06.2012, 2 우 우 e.l. 09.06.2012; 47 Säcke 09.06.2014: Tsada; 4 Säcke am 25.03.1999: Corallia Beach; 5 Säcke 28.03.1999, 1 Sack 13.03.2012, 5 Säcke 19.03.2012: Petra tou Rõmiou; 2 Säcke 12.03. 
2012: Mandria N bei Paphos; 1 Sack 12.03.2012: Kouklia 2 km SE; 3 Säcke 13.03.2012: Kouklia 2 km NW; 1 Sack 14.03.2012: Nea Dimmata; 8 Säcke 14.03.2012: Panagia Galaterousa; 1 Sack 14.03.2012: Pomos; 1 Sack 15.03. 2012: Paramali; 1 Sack 15.03.2012: Apollon Hylátis Heiligtum; 1 Sack 15.03.2012: Antikes Stadion Koúrion; 4 Säcke 15.03.2012: Autobahnabfahrt Moni; 1 Sack 15.03.2012, 7 Säcke 10.06.2014: Governor's Beach; 2 Säcke 15.03.2012: Stavrovouni Monastery; 1 Sack 16.03.2012: Souni; 1 Sack 17.03.2012: Avakas-Schlucht; 7 Säcke 10.06.2014: Cavo Greko; 2 Säcke 10.06.2014: Liopetri; 5 Säcke 10.06.2014: Pano Kivides; 1 Sack 12.06. 2014: Pano Lefkara; 1 Sack 13.06.2014: Episkopi; 1 Sack 14.06.2014: Saittas; 1 Sack 14.06.2014: Omodus; 1 Sack 15.06.2014: Pelentri, alles leg. WeIDLICH.

Diskussion: Umfangreiche Aufsammlungen und Züchtungen sowie vergleichende Untersuchungen haben bestätigt, dass es sich hierbei um die Nominatform von P. villosella O. handelt. Insbesondere der Flügelschnitt der Männchen, der Aderungsverlauf der Flügel und die Form der Diskoidalzelle ähneln sich weitgehend. Lediglich ein etwas gestreckterer Vorderflügelschnitt ist den Populationen der Republik Zypern eigen (Fig. 4c). Die Nominatform-Population von Yilanlikale weist dagegen einen mehr gerundeten Vorderflügelschnitt auf (Fig. 4b). Dass es sich in beiden Fällen um das gleiche Taxon handelt, wurde durch mitochondiale DNA - Untersuchungen im Rahmen Barcode of Life Data Systems (iB0L) der Universität of Guelph, Ontario, Kanada untersetzt. Hierzu wurden $2 \sigma^{\star}$ aus Tsada (Republik Zypern - Fundort 7; BC TLMF Lep 07777 und 07778, e.l. 12.05.1999, leg. Weiduich) mit $1 \sigma^{\star}$ von Yilanlikale aus den Nurdaği (Türkei - Kilikien; BC TLMF Lep 07076, e.1. 09.05.2010, leg. WeIDLICH) verglichen. Das Ergebnis zeigt dass beide quasi genetisch identisch sind.

Weitere molekulare Untersuchungen weisen folgende Ergebnisse auf: Die o.g. zypriotischen und türkischen Populationen unterscheiden sich von denen aus Griechenland (Makedonia - Katerini $=$ BC TLMF Lep 07773, 1 o e.l. 15.-26.05.2007, leg. Weiduich und Ionische Inseln, Ithaka - Levki $=$ BC TLMF Lep 07774, $1 \sigma^{\star}$ e.l. 11.05.2003, leg. Weidlich) um mehr als $5 \%$. Ähnliche hohe Differenzen zeigt bereits HätTENSCHWILER (2011: 176) auf, der P. villosella-Populationen aus Griechenland (Peloponnes-Sparta) mit denen der ssp. quadratica De FreinA, 1983 aus der Südtürkei (KilikienKozan) vergleicht und genetische Differenzen von 6,67 \% feststellt.

Interessant in diesem Zusammenhang ist aber auch, dass die genetischen Untersuchungen der Population der ssp. quadratica aus der Südtürkei ( $1 \sigma^{\star}$, Lykien - Belbidi bei Antalya, e.l. Mitte 07.2007, leg. WeIDLICH) im Vergleich zu denen der vermeintlich zur Nominatform gehörenden aus Tsada und Yilanlikale lediglich eine Differenz von nur 0,3\% aufweist. Die morphologischen Merkmale, die bei der Beschreibung dieser Unterart herangezogen wurden (Männchen), wie eine andere Gestalt der Diskoidalzelle (distale Ausbildung) und ein anderer, gerundeter Flügelschnitt lassen sich somit bisher nicht ausreichend interpretieren (siehe Fig. 4a-d). Einzig und allein die Beschuppung der Kammzähne an den Fühlern der $o^{x} o^{x}$ ist ein klar erkennbares, differentes morphologisches Detail bei ssp. quadratica.

P. villosella villosella verfügt über sehr vielgestaltige Sackformen (Fig. 5). Die unterschiedliche Gestalt und Ausprägung der Säcke von quadratica zu denen der Nominatform, wie HäTtenschwiler (2011: 173-176) mitteilt, liegen in der Variationsbreite. Sie sind z.T. auch den Zuchtbedingungen geschuldet und können somit nicht für die Aufrechterhaltung eines gesonderten Taxons dienen.

Die vorliegenden Genitaluntersuchungen zeigen, dass es nur sehr geringe Unterschiede im Bereich der Anelli und Clasper sowie des Tegumendaches, Vinculums und des Saccus gibt. Auch Form und Ausbildung des Aedaeagus ist weitgehend ähnlich. Insgesamt gesehen können die Populationen der Nominatform und der ssp. quadratica genitaliter kaum voneinander unterschieden werden (Fig. 6).

Zusammenfassend kann somit dargestellt werden, dass sich die beispielhaft untersuchten griechischen Populationen, die vermutlich der Nominatform villosella angehören, von den morphologisch ähnlichen villosella aus der Republik Zypern und der Südtürkei genetisch deutlich zwischen 5 und 6,67 \% unterscheiden. Dagegen gibt es so gut wie keine erkennbaren genetischen Unterschiede zwischen der ssp. quadratica aus der Südtürkei und den Populationen aus der Südtürkei und der Republik Zypern, die morphologisch der Nominatform zuzurechnen sein dürften. Im Ergebnis führt dies zu der Feststellung, dass der taxonomische Status von quadratica allein durch ein morphologisches Merkmal, nämlich die beschuppten Kammzähne der männlichen Antennen, bisher aufrecht erhalten werden kann.

Die Ausführungen zu den verschiedenen Populationen von P. villosella aus Griechenland, der Türkei und der Republik Zypern zeigen auf, dass in diesem Komplex eine uneingeschränkte Nutzbarkeit bzw. Interpretationsfähigkeit von morphologischen einerseits und genetischen Merkmalen andererseits hinsichtlich der Abgrenzung einzelner Populationen und ihrer Zugehörigkeit zu verschiedenen Taxa kaum möglich ist.

In der Republik Zypern kommt P. villosella villosella verbreitet vor und kann stellenweise sehr häufig auftreten. So wurden z.B. 2012 in der Umg. Tsada ca. $3 \mathrm{~km} \mathrm{~N}$, 450 m NN über 100 Säcke gezählt (grösstenteils vorjährige, alte Säcke).

Fischer \& LEWANDOWSKI (2003: 301) erwähnen den Schlupf eines $1 \sigma^{*}$ am 29.02.1999 und der Autor selbst züchtete die $\sigma^{*} o^{*}$ und +9 o zwischen dem 13.04. und 
12.06. Die Art besiedelt unterschiedliche Lebensräume und wurde ausschließlich in Offenlandschaften hauptsächlich auf Kalkgesteinen und hier vorrangig am Fuß von Kalkabbrüchen beobachtet. In der Höhenverbreitung kommt die Art auf Zypern zwischen Meeresspiegelniveau und $900 \mathrm{~m} \mathrm{NN}$ vor.

\section{Apteronini TUTT, 1900}

\section{Apterona helicoidella (VALLOT, 1827)}

Untersuchtes Material: 1 Sack 07.03.1998: Neo Chõrio; 1 Sack 07.03.1998, 2 Säcke 25.03.1999: Páfos; i.A. Säcke 09.03.1998, i.A. Säcke 25.03.1999, i.A. Säcke 14.03.2012, i.M. Säcke 09.06.2014: Tsada; 15 Säcke 10.03.1998: Kykko-Monastery 7 km E; 2 Säcke am 25.03.1999 Corallia Beach; 1 Sack 26.03.1999: Koilani; i.M. Säcke 28.03.1999, i.M. Säcke 13.03.2012, i.A. Säcke 07.06.2014: Petra tou Rõmiou; i.M. Säcke 28.03.1999: Parekklisia S; i.M. Säcke 28.03.1999: Kellaki; 1 Sack 28.03.1999: Odou; 1 Sack 28.03.1999: Apliki; i.A. Säcke 12.03.2012: Mandria N bei Páfos; i.M. Säcke 12.03.2012: Kouklia 2 km SE; i.M. Säcke 12.03.2012: Columbia Beach; 2 Säcke 13.03.2012: Kouklia 2 km NW; 1 Sack 13.03.2012: Kidasi; i.M. Säcke 14.03.2012: Goudi; i.M. Säcke 14.03.2012: Panagia Galaterousa; i.M. Säcke 15.03.2012: Paramali; i.M. Säcke 15.03.2012: Apollon Hylátis Heiligtum; i.M. Säcke 15.03.2012: Autobahnabfahrt Moni; i.A. Säcke 15.03.2012, i.M. Säcke 10.06.2014: Governor's Beach; i.A. Säcke 15.03.2012: Kalo Chõrio; i.A. Säcke 15.03.2012: Stavrovouni Monastery; 1 Sack 15.03.2012: Lythrodontea; 1 Sack 16.03.2012: Alassa; 1 Sack 16.03.2012: Monagri; i.M. Säcke 16.03.2012: Pera Pedi; 1 Sack 16.03.2012, i.M. Säcke 07.06.2014: Souni; i.M. Säcke 09.06.2014: Mandria; i.M. Säcke 12.06.2014: Evrichou; i.A. Säcke 12.06.2014: Koronia; 1 Sack 12.06.2014: Pano Lefkara; 1 Sack 12.06.2014: Dhoros; i.M. Säcke 14.06.2014: Omodus; i.M. Säcke 15.06.2014: Kyperounta; 2 Säcke, 15.06.2014: Palaichori, alles leg. WEIDLICH.

Diskussion: Diese Psychidenart ist in der Republik Zypern weit verbreitet und kommt hier in ihrer parthenogenetischen Form vor. Stellenweise wurde sie sehr häufig angetroffen und so konnten in der Umgebung von Petra tou Rõmiou am 28.03.1999 und bei Pera Pedi NW am 16.03.2012 hunderte Säcke an Leitplanken beobachtet werden. An einem Strommast beim Hotel Columbia Beach fanden sich am 12.03.2012 über 80 Säcke.

\section{Danksagung}

Meinem Freund W. ArnscheId (Bochum/Deutschland) sei an dieser Stelle für seine Unterstützung bei den Diskussionen und Korrekturen sowie für die Anfertigung der Genitalpräparate und deren Fotografie herzlich gedankt.

\section{Literatur}

Arenberger, E. 1994: Zusammenfassende Darstellung der Mikrolepidopterenfauna Zyperns. - Annales Musei Goulandris 9: 253-336.

Arenberger, E. \& Wimmer, J. 2003: Dritter Nachtrag zur Mikrolepidopterenfauna Zyperns. - Quadrifina 6: 43-54.

Arnscheid, W. R. \& Weidlich, M. 2015: Microlepidoptera of Europe. - Psychidae. - in Vorbereitung.

DANiEL, F. 1964: Die Lepidopterenfauna Jugoslawisch Mazedoniens. II. Bombyces et Sphinges. - Prirodonaučen Muzej Skopje, Posebno izdanie Nr. 2: 1-74.

De FreinA, J. J. 1983: Beitrag zur systematischen Erfassung der Bombyces- und Sphinges-Fauna Kleinasiens. Neue Kenntnisse über Artenspektrum, Systematik und Nomenklatur sowie Beschreibung neuer Taxa (Lepidoptera). - Mitteilungen der Münchner Entomologischen Gesellschaft 72: 57-127.

De Freina, J. J. 1994: 9. Beitrag zur systematischen Erfassung der Bombyces- und Sphinges-Fauna Kleinasiens. Weitere Kenntnisse über Artenspektrum, Systematik und Verbreitung von Cossidae, Psychidae, Cochlididae, Syntomidae, Saturniidae, Brahmaeidae, Drepanidae, Axiidae, Hepialidae, Dilobidae und Nolidae (Insecta, Lepidoptera). - Atalanta 25 (1/2): 317-349.

Fischer, H. \& LewANDowski, S. 2003: Zweiter Beitrag zur Schwärmer- und Spinnerfauna von Zypern (Lepidoptera). - Entomologische Zeitschrift 113 (10): 295-301.

Fischer, H. \& LewANdowski, S. 2010: Aktualisierte Checkliste der Geometridenarten von Zypern inklusive der wichtigsten Literaturangaben zu dieser Familie (Lepidoptera, Geometridae). - Atalanta 41 1/2: 265-269, Marktleuthen.

HätTenschwiler, P. 1998: Neue Eumasia Arten aus Mittelspanien und den Malediven und einige Ergänzungen zur Kenntnis der Gattung Eumasia (Psychidae). - Nota lepidopterologica 21 (4): 264-282.

Hättenschwiler, P. 2011: Lebensweise und BeschreibungderEntwicklungsstadienvon Pachythelia villosella quadratica DE FreInA, 1983 (Psychidae: Oiketicinae: Acanthopsychini). - Nota lepidopterologica 34 (2): 171-177.

Hausmann, A. 1995: Neue Geometriden-Funde aus Zypern und Gesamtübersicht über die Fauna. Mitteilungen der Münchner Entomologischen Gesellschaft 85: 79-111.

Karsholt, O. \& Nieukerken, E. J. van 2013: Lepidoptera. Moth. Fauna Europaea, version 2.6 .2 - http// www.faunaeur.org/distribution.php.

LEDERER, J. 1855: Beitrag zur Schmetterlings-Fauna von Cypern, Beirut und einem Theile Klein-Asiens. - Verhandlungen der zoologisch-botanischen Gesellschaft in Wien 5: 177-254. 
LEWANDOWsKi, S. 2000: Beitrag zur Lepidopterenfauna von Zypern. - Entomologische Zeitschrift 110 (12): 376-377.

Lewandowski, S. \& Fischer, K.-H. 2002: Beitrag zur Schwärmer- und Spinnerfauna von Zypern, sowie eine Übersicht der bisher bekannten Arten (Lepidoptera) - Entomologische Zeitschrift 112 (9): 264-272.

Lewandowski, S. \& Fischer, H. 2004: Check-Liste der Noctuidae von Zypern. - Atalanta 35 (1/2): 119-126.

LewANDowski, S. \& MAKRIs, C. 2006: Dritter Beitrag zur Fauna der „Spinner und Schwärmer“ Zyperns: Neue Erkenntnisse zur Gattung Ocneria HüBNER, 1819 (Lepidoptera, Lymantriidae) und aktualisierte Daten $\mathrm{zu}$ anderen Familien. - Nachrichten entomologischer Verein Apollo, N. F. 27 (3): 165-170.

MAKris, C. 2003: Butterflies of Cyprus. - 329 pp., Bank of Cyprus Cultural Foundation, Nicosia.

Rebel, H. 1916: Ueber die Lepidopterenfauna Cyperns. - XXVI. Jahres=Bericht des Wiener entomologischen Vereines: 93-110 (1915).

Rebel, H. 1939: Zur Lepidopterenfauna Cyperns. - Mitteilungen der Münchner entomologischen Gesellschaft 29: 487-564.

Sauter, W. 1956: Morphologie und Systematik der schweizerischen Solenobia-Arten. - Revue Suisse de Zoologie 63 (27): 451-550.

Soвсzyк, T. 2011: World Catalogue of Insects. Volume 10. Psychidae (Lepidoptera). - 467 S., Apollo Books, Stenstrup.
WeIdlich, M. 2010: Eumasia parietariella (HeydenREICH, 1851), erstmals in der Slowakei nachgewiesen (Lepidoptera: Psychidae). - Entomologische Zeitschrift 120 (3): 129-131.

Weidlich, M. 2013a: Zur Psychidenfauna Albaniens unter Berücksichtigung der Albanien-Expedition 1961 des Deutschen Entomologischen Institutes (Lepidoptera: Psychidae). - Beiträge zur Entomologie 63 (2): 325-338.

WeIDLICH, M. 2013b: The bisexual Dahlica triquetrella (HüBNER, 1813) and new records of Luffia lapidella (Goeze, 1783) in Slovenia (Lepidoptera: Psychidae). - Acta Entomologica Slovenica 21 (2): 163-168.

Weidlich, M. \& Henderickx, H. 2002: Eine neue Pseudobankesia aus Zypern (Lepidoptera Psychidae). - Linzer biologische Beiträge 34 (1): 503-512.

Wimmer, J. 1985: Beitrag zur Macrolepidopterenfauna von Zypern. - Jahresberichte Steyrischer Entomologen 1985: 54-61.

Witt, T. J. 1984: Neue und bemerkenswerte Heterocerennachweise aus Griechenland (Lepidoptera, Arctiidae, Lymantriidae, Thyatiridae, Psychidae). Nachrichtenblatt bayerischer Entomologen $34(2)$ : 47-49.

Zagulyajev, A. K. 1996: New and little known species of moth (Lepidoptera: Psychidae, Tineidae, Pterophoridae, Alucitidae) of the fauna of Russia and neighbouring territories IX. - Entomological review 75 (1): 117-131.

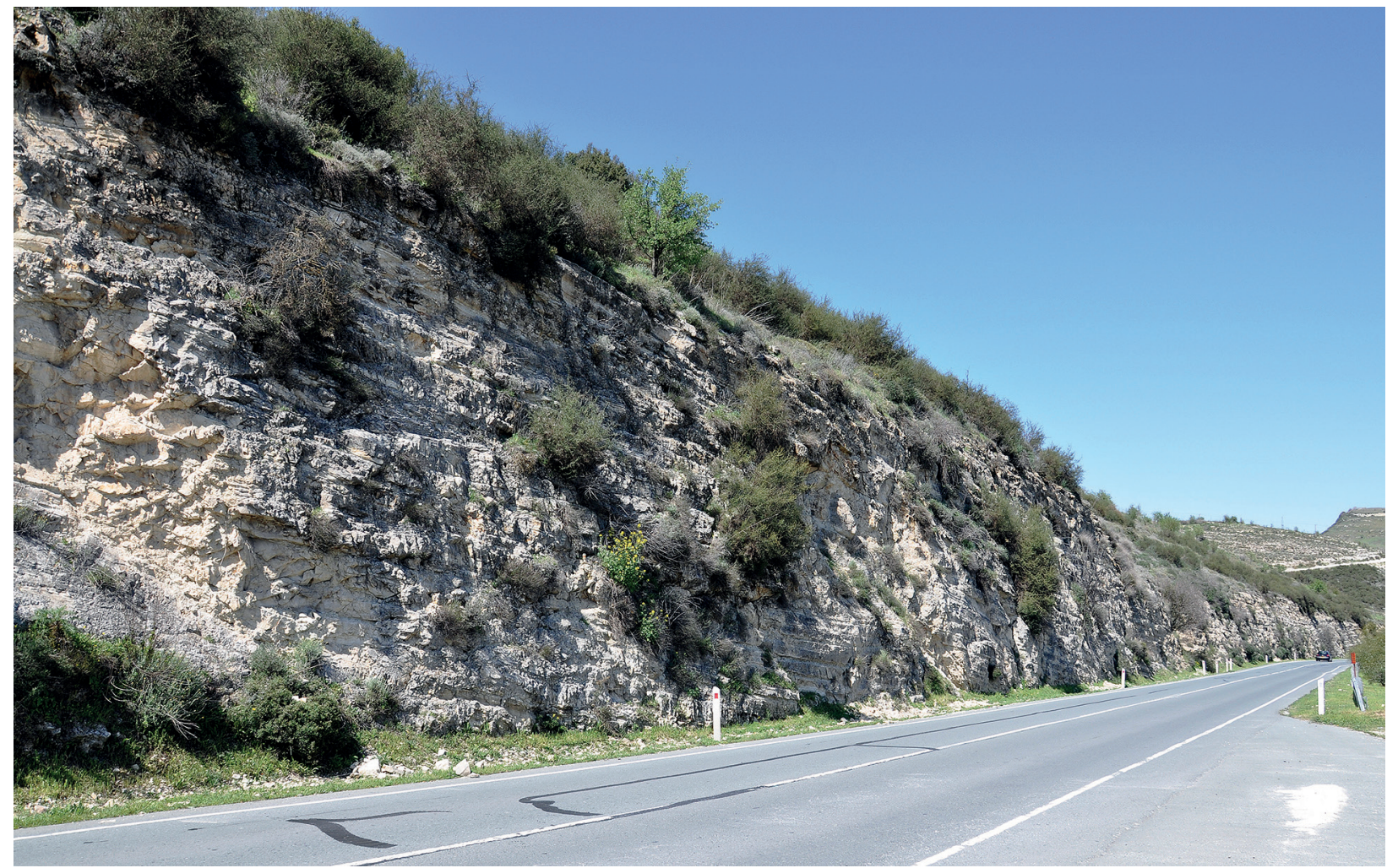

Fig. 3: Umg. Páfos, Umg. Tsada N, Lebensraum von Eumasia cyprianella spec. nov. (Foto: 17.03.2012, M. WeIDLICH). 


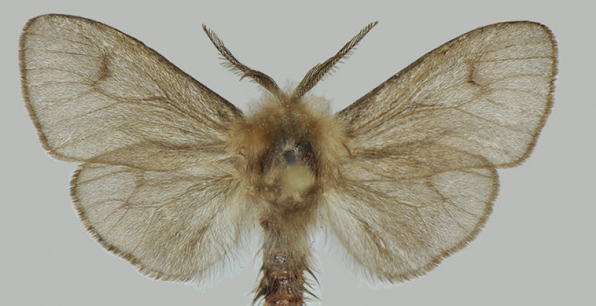

a

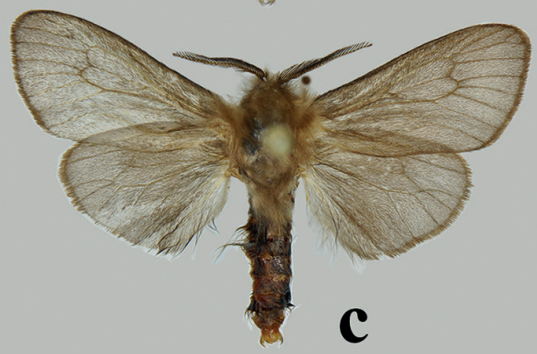

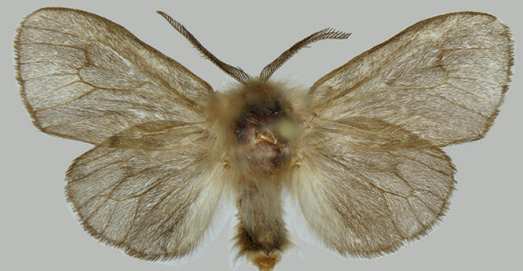

b

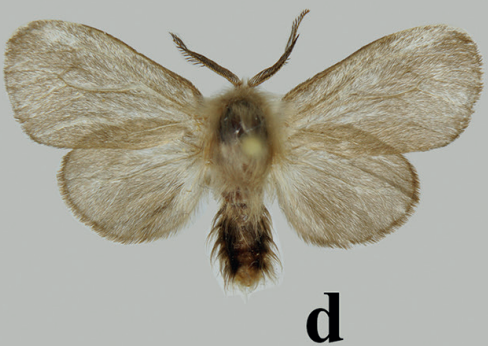

Fig. 4: $\sigma^{\Uparrow}-$ P. villosella: a) e.l. 11.05.2004 (Flügelspanne 24,5 mm), Asia occidentalis, Türkei, Akcakoca Daglari, Karaman SE Zonguldak, b) e.l. 15.05.2010, Asia occidentalis, Türkei, Kilikien, Nurdaği, Yilanlikale bei Ceyhan, c) e.l. 03.05.1999, Asia occidentalis, Republik Zypern, Umg. Páfos, Umg. Tsada $3 \mathrm{~km} \mathrm{N,} \mathrm{P.} \mathrm{villosella} \mathrm{quadratica:} \mathrm{d)} \mathrm{e.l.}$ 29.05.2010, Asia occidentalis, Türkei, Pamphylien, Konakli bei Alanya, alles leg. Weiduich. (Foto: M. WeIdLICH).

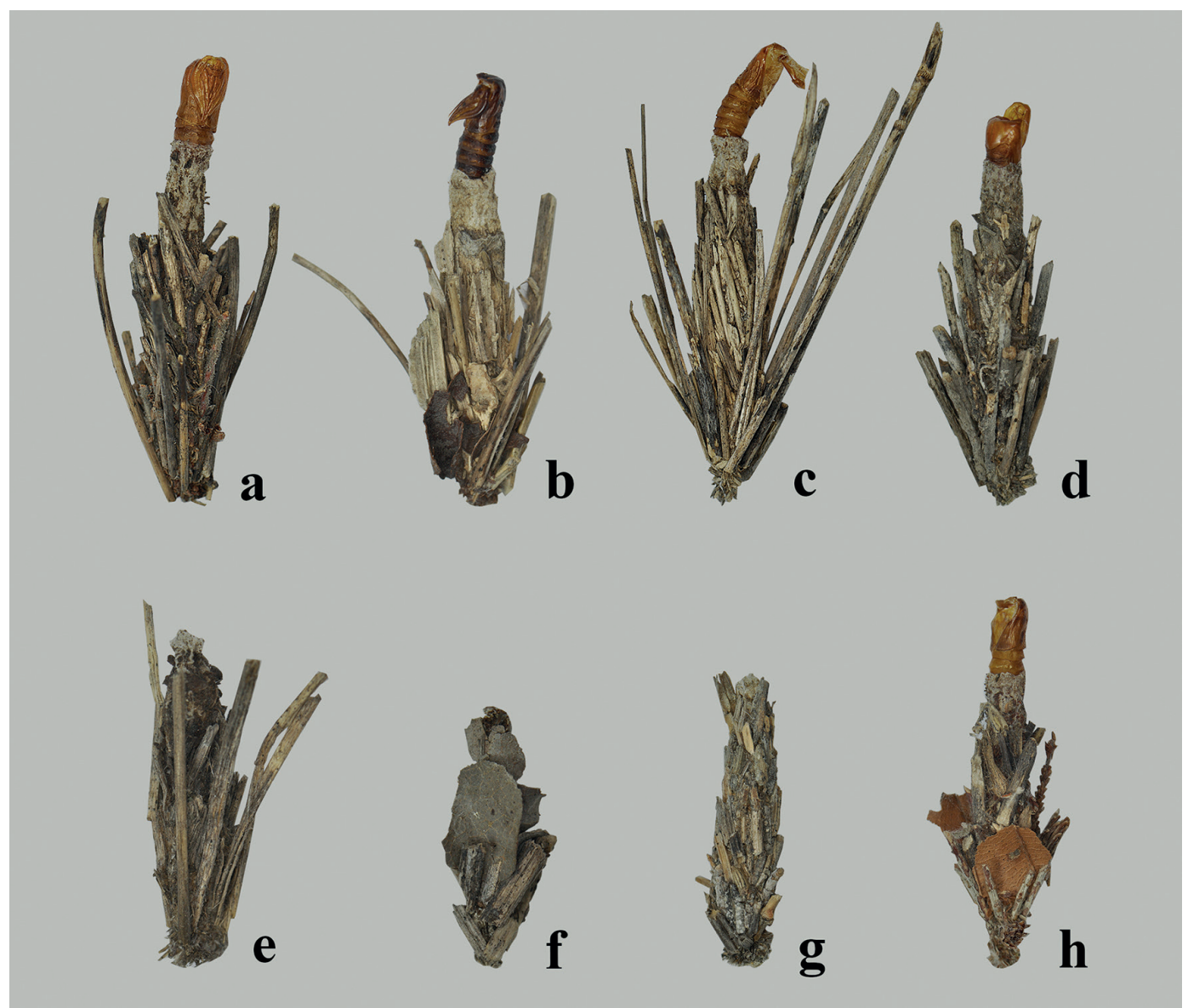

Fig. 5: Säcke - P. villosella: a) e.l. 20.-30.05.2007 (Sacklänge mit Puppenhülle $40 \mathrm{~mm}$ ), Europa meridionalis, Griechenland, Makedonien, Óros Pangéo, Akrovouni S Elefteropouli, b) e.l. 11.05.2004, Asia occidentalis, Türkei, Akcakoca Daglari, Karaman SE Zonguldak, c) e.l. 15.05.2010, Asia occidentalis, Türkei, Kilikien, Nurdaği, Yilanlikale bei Ceyhan, d) e.l. 03.05.1999, Asia occidentalis, Republik Zypern, Umg. Páfos, Umg. Tsada 3 km N, e) 09.06.2014, Asia occidentalis, Republik Zypern, Umg. Páfos, Umg. Tsada 3 km N, f, g) 09.06.2014, Asia occidentalis, Republik Zypern, Umg. Páfos, Umg. Tsada $3 \mathrm{~km} \mathrm{~N}$, P. villosella quadratica: h) e.l. 29.05.2010, Asia occidentalis, Türkei, Pamphylien, Konakli bei Alanya, alles leg. Weidlich. (Foto: M. Weiduich). 


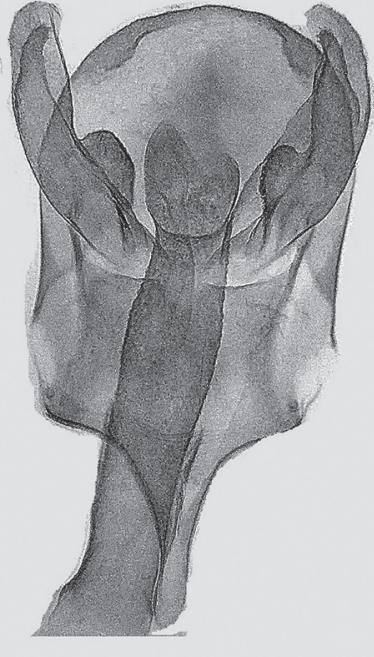

a

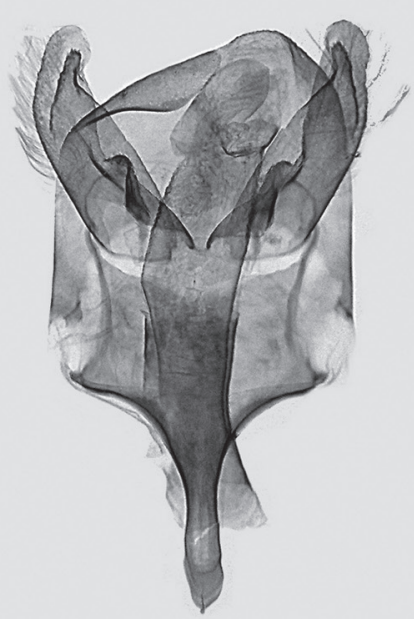

d

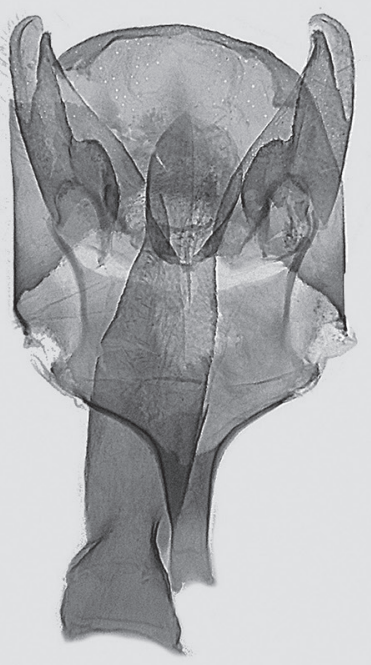

b

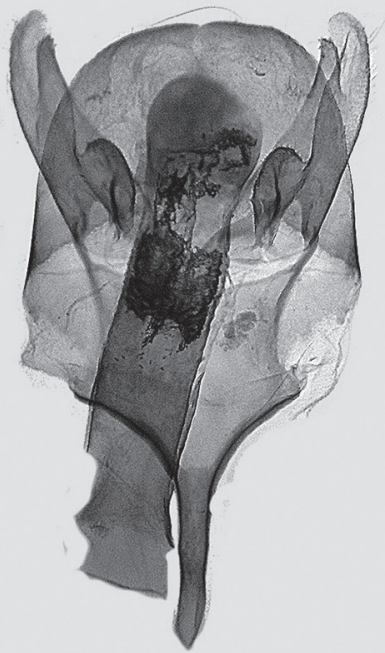

e

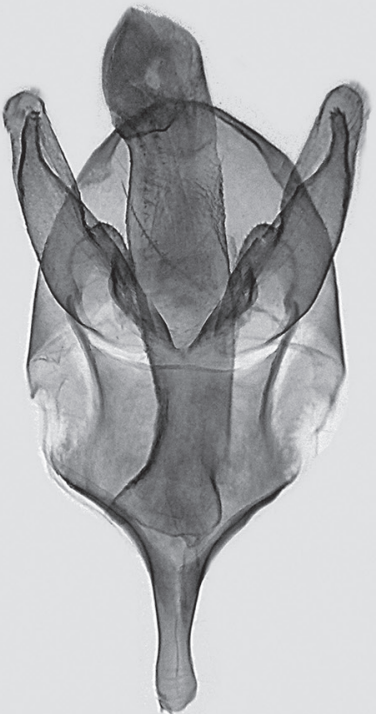

C

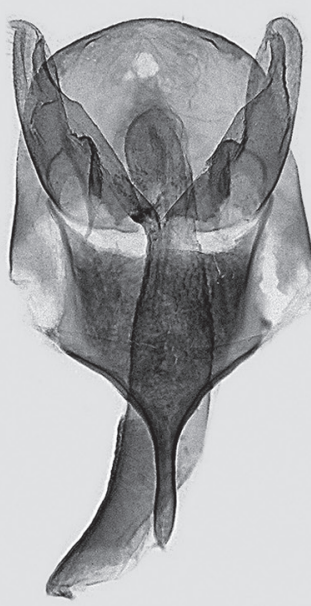

f

Fig. 6: $\sigma^{\top}-$ Genital P. villosella. a) Europa meridionalis, Italien, Provinz Imperia, Chiappa, Lichtfang 13.-25.06.1984, leg. W. Arnscheid, b) e.l. 20.-30.05.2007, Europa meridionalis, Griechenland, Makedonien, Óros Pangéo, Akrovouni S Elefteropouli, c) e.l. 11.05.2004, Asia occidentalis, Türkei, Akcakoca Daglari, Karaman SE Zonguldak, d) e.l. 15.05.2010, Asia occidentalis, Türkei, Kilikien, Nurdagi, Yilanlikale bei Ceyhan, e) e.l. 03.05.1999, Asia occidentalis, Republik Zypern, Umg. Páfos, Umg. Tsada 3 km N, P. villosella quadratica. f) e.l. 29.05.2010, Asia occidentalis, Türkei, Pamphylien, Konakli bei Alanya, alles leg. WeIDLICH (Fotos: W. ARNSCHEID). 